\title{
-NOTES-
}

\section{A SIMPLIFIED GEOMETRIC PROOF OF THE RECIPROCAL STRESS THEOREM*}

\author{
BY B. PAUL (Brown University)
}

The well-known reciprocal stress theorem $[1,2]^{1,2}$ states that the scalar product of the stress vector on a given plane with the unit normal to a second plane equals the scalar product of the stress vector on the second plane with the unit normal to the first plane. Starting from this single theorem, which Biezeno and Grammel [1] call "the essential core of all stress analysis" it is a simple matter to deduce the symmetry of the stress tensor, to derive the equations of stress transformation, and to find many other useful results.

Biezeno and Grammel prove the theorem geometrically by projecting the faces of a stressed tetrahedron and their corresponding stress vectors orthogonally onto an auxiliary plane. This is essentially a proof by a technique of descriptive geometry. The importance of the theorem may perhaps justify the small amount of space required here to present a simpler modified version of this proof which obviates the need for projections onto an auxiliary plane.

For simplicity consider a homogeneous state of stress where $S_{1}$ and $S_{2}$, respectively, represent the stress vectors on planes $\Pi_{1}$ and $\Pi_{2}$ which are respectively normal to unit vectors $\mathrm{n}_{1}$ and $\mathrm{n}_{2}$. Figure 1 shows two congruent triangles $A B C$ and $A B D$ constructed. on the line of intersection of $\Pi_{1}$ and $\Pi_{2}$. The four points $A B C D$ enclose a tetrahedron. Let $E, F, G, H$ represent the centroids of the four faces as shown in Fig. 1 . Then it follows that the plane of $E G H$ is parallel to $\Pi_{2}$ and the plane of $F G H$ is parallel to $\Pi_{1}$.

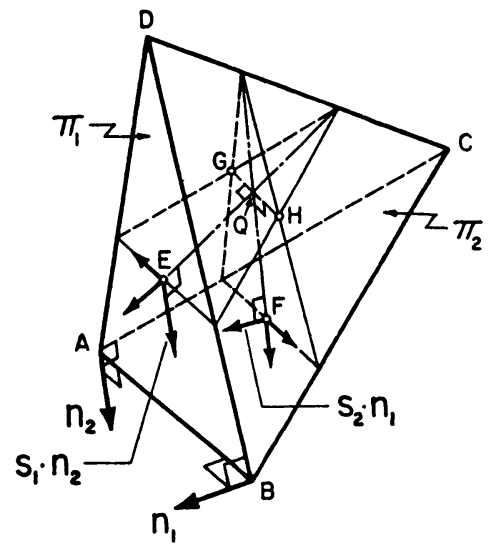

Fig. 1.

*Received Oct. 19, 1959

'C. B. Biezeno and R. Grammel, "Engineering Dynamics, Vol. I," p. 3, Blackie, 1955.

2I. S. Sokolnikoff, "Mathematical Theory of Elasticity," 2nd Ed., p. 43, McGraw-Hill, 1956. 
The resultant force vectors on faces $B C D$ and $A C D$ act at the centroids $H$ and $G$ of these faces and therefore contribute nothing to the sum of moments about axis $G H$. Denoting the mid-point of $G H$ by $Q$, decompose the stress vector $\mathrm{S}_{1}$ acting at $E$ into three components that are acting along $Q E$, parallel to $Q H$, and normal to the plane $E G H$ (i.e., parallel to $\mathbf{n}_{2}$ ), respectively. Of these only the last component, which has the value $S_{1} \cdot \mathbf{n}_{2}$, produces a moment about axis $G H$. Similarly, only the component of $\mathrm{S}_{2}$, acting at $F$, in the direction of $\mathrm{n}_{1}$, produces a moment about axis $G H$. The resultant moment is given by

$$
\left(\mathbf{S}_{1} \cdot \mathbf{n}_{2}\right) A(E Q)-\left(\mathbf{S}_{2} \cdot \mathbf{n}_{1}\right) A(F Q)=0 .
$$

In the above equation $A$ is the common area of triangles $A B C$ and $A B D$. Since the moment arms $E Q$ and $F Q$ are equal by construction, it follows that

$$
S_{1} \cdot n_{2}=S_{2} \cdot n_{1} \text {. }
$$

\section{UNSTEADY MOTION OF AN INFINITE LIQUID DUE TO THE UNIFORM ROTATION OF A SPHERE, $r=a^{*}$ \\ BY C. D. GHILDYAL (Lucknow University)}

Introduction. The problem of steady motion of a viscous liquid due to the slow rotation of a sphere has already been discussed by various workers in the field of hydrodynamics [1]. Here we propose to discuss the unsteady flow of a liquid initially at rest due to the uniform rotation, $\Omega$, of a sphere about the axis of $z$, under an external force $z \omega^{2}$ acting per unit mass of the liquid along the axis of rotation and the pressure at any point of the liquid is given by

$$
p=\int \rho r \omega^{2} d r+a \text { constant, }
$$

where $\omega \equiv \omega(r, t), r^{2}=x^{2}+y^{2}+z^{2}$, and velocity components at any point $(x, y, z)$ of the liquid are assumed to be $u=-\omega y, v=\omega x$ and $w=0$.

The result obtained is valid also for slow rotation of the sphere. In that case the external force does not exist and the pressure remains constant throughout the liquid.

The equations of motion of a viscous homogeneous incompressible liquid in this case reduce to

$$
\begin{aligned}
& \frac{\partial \omega}{\partial t}=\nu\left(\frac{\partial^{2} \omega}{\partial r^{2}}+\frac{4}{r} \frac{\partial \omega}{\partial r}\right), \\
& \frac{\partial p}{\partial r}=\rho r \omega^{2} .
\end{aligned}
$$

A general solution of $(B)$ is obtained by the method of Laplace transform under the boundary conditions

$$
\begin{array}{ll}
\text { (i) } & \omega(a, t)=\Omega, \\
\text { (ii) } & \omega(r, 0)=0, \\
\text { (iii) } & \lim _{r \rightarrow \infty} \omega(r, t)=0 .
\end{array}
$$

*Received August 26, 1959; revised manuscript received January 20, 1960. 